\title{
Avaliação da mamada, autoeficácia do aleitamento materno e fatores influentes no desmame precoce em primíparas
}

\section{Breastfeeding assessment, self-efficacy of breastfeeding and influential factors in early weaning in primiparous women}

Evaluación de la lactancia materna, autoeficacia de la lactancia materna y factores influyentes en el destete temprano en mujeres primíparas

Geisilene da Conceição Costa ${ }^{1}$ Delia Esmeyre Paredes ${ }^{2}$ Silvio Assis de Oliveira Junior ${ }^{3}$ Ana Beatriz Gomes de Souza Pegorare ${ }^{4}$

${ }^{1}$ Fisioterapeuta do Programa de Residência Multiprofissional em Cuidados Continuados Integrados (CCl) - Hospital São Julião e Universidade Federal de Mato Grosso do Sul. E-mail: geisilene.costa@gmail.com, Orcid: http://orcid.org/0000-0003-4054-1437

2 Enfermeira do Hospital Universitário Maria Aparecida Pedrossian (HUMAP). E-mail: delia.smeire@gmail.com, Orcid: http://orcid.org/0000-0003-3440-6589

${ }^{3}$ Docente do Programa de Pós-Graduação em Ciências do Movimento da Universidade Federal de Mato Grosso do Sul. E-mail: silvio.assis@gmail.com, Orcid: http://orcid.org/0000-0002-8895-9456

${ }^{4}$ Docente do Programa de Pós-Graduação em Ciências do Movimento da Universidade Federal de Mato Grosso do Sul.E-mail: anabegs@hotmail.com, Orcid: http://orcid.org/0000-0002-6958-5719 
Resumo: O leite materno é o alimento mais recomendado para o bebê, porém as taxas de aleitamento materno no Brasil não atingiram níveis desejáveis. Objetivou-se avaliar a confiança das primíparas no aleitamento materno; identificar os parâmetros desfavoráveis à amamentação e correlacioná-los ao desmame precoce. Pesquisa prospectiva e observacional, composta por 101 primíparas avaliadas por meio da Escala de Autoeficácia da Amamentação (EAAM) e do Formulário de Observação da Mamada (FOM), no período pós-parto imediato e posterior, via contato telefônico, a fim de informar sobre a continuidade do aleitamento materno e/ou desmame. Elevados escores de EAAM foram encontrados, possivelmente, por conta do suporte da equipe que atua na promoção do aleitamento materno. A observação da mãe constituiu a variável preditiva para ocorrência do desfecho com desmame precoce. Comportamentos preditivos para o desmame precoce estão associados à condição física da mãe durante a mamada, sendo fundamental o monitoramento dos parâmetros desfavoráveis à amamentação, pela equipe.

Palavras-chave: aleitamento materno, período pós-parto, mães.

\begin{abstract}
Although breast milk is the normative feeding for infants, breastfeeding rates are lower than recommended. This study aimed to evaluate the confidence of primiparous women in breastfeeding; identify unfavorable breastfeeding parameters and correlate them with early weaning. Prospective and observational research, consisting of 101 primiparous women assessed by the Breastfeeding Self-Efficacy Scale (BSES) and Breastfeeding Observation Form (BOF) in the immediate and subsequent postpartum period, via telephone contact, to inform continuity breastfeeding and/or weaning. High BSES scores were found, possibly due to the support of the team that promotes breastfeeding. Observation of the mother was the predictive variable for the occurrence of early weaning outcome. Predictive behaviors for early weaning are associated with the mother's physical condition during breastfeeding. The monitoring of unfavorable breastfeeding parameters by the team is essential.
\end{abstract}

Keywords: breastfeeding, postpartum period, mothers.

Resumen: La leche materna es la alimentación normativa para los lactantes, pero las tasas de lactancia materna en Brasil no han alcanzado niveles deseables. Este estudio tuvo como objetivo evaluar la confianza de las mujeres primíparas en la lactancia materna; identificar parámetros de lactancia desfavorables y correlacionarlos con el destete temprano. Investigación prospectiva y observacional, que consta de 101 mujeres primíparas evaluadas por la Escala de Autoeficacia de la Lactancia Materna (EAAM) y el Formulario de Observación de la Lactancia Materna (FOM) en el período posparto inmediato y posterior, vía contacto telefónico, para informar la continuidad lactancia materna y/o destete. Se encontraron puntuaciones altas de EAAM, posiblemente debido al apoyo del equipo que promueve la lactancia materna. La observación de la madre fue la variable predictiva para la aparición de resultados de destete temprano. Los comportamientos predictivos para el destete temprano están asociados con la condición física de la madre durante la lactancia. El monitoreo de los parámetros desfavorables de lactancia por parte del equipo es esencial.

Palabras clave: lactancia materna, posparto, madres. 
Avaliação da mamada, autoeficácia do aleitamento materno e fatores influentes no desmame precoce em primíparas

\section{INTRODUÇÃO}

O aleitamento materno (AM) é um processo que envolve não apenas a alimentação do recém-nascido, mas a formação de um profundo vínculo afetivo entre mãe e bebê, gerando benefícios para ambos. O leite humano, além de ser nutricionalmente completo, também possui vários fatores de proteção que ajudam a reduzir as internações por pneumonia em crianças menores de seis meses em aleitamento materno exclusivo e, em cerca de $50 \%$, em crianças de 9 a 12 meses.

Estima-se que o AM tenha o potencial de reduzir em $13 \%$ a mortalidade infantil por causas evitáveis, especificamente em menores de cinco anos, sendo esta uma meta a ser atendida por um dos Objetivos de Desenvolvimento Sustentável, no período de 2016 a 2030.

Apesar dos esforços globais com a implementação de programas e políticas públicas para o incentivo ao AM, as taxas de início precoce, duração e exclusividade ainda não atingiram níveis desejáveis. Sabe-se que um dos aspectos responsáveis pelo desmame precoce está associado aos fatores socioculturais, aspectos intrínsecos à mulher, características anátomo-fisiológicas e, especialmente, às dificuldades iniciais no manejo do processo da amamentação.

Nesta perspectiva, diante dos inúmeros fatores que interferem no aleitamento materno exclusivo (AME), as dificuldades iniciais, embora pouco estudadas, podem estar entre as principais facilitadoras do desmame precoce e podem fazer com que as mães sejam menos propensas a amamentar um segundo filho. Sendo assim, o Fundo das Nações Unidas para a Infância (UNICEF) propôs um protocolo de observação que possibilita a identificação e o rastreamento das principais dificuldades associadas. Embora o instrumento seja útil, são escassos os registros na literatura sobre o seu uso. Sem uma avaliação cuidadosa da técnica da amamentação, os estudos tendem a subestimar as dificuldades iniciais associadas a este processo. O uso deste instrumento na prática clínica poderia qualificar a comunicação escrita entre os profissionais da equipe que trabalham no manejo do aleitamento materno e, ainda, facilitar a proposta de condutas individualizadas ao binômio mãe-bebê, além de atuar como um indicador de qualidade nos serviços de saúde materno-infantil. 
Portanto o presente estudo teve por objetivo investigar a confiança das primíparas no aleitamento materno, identificar os sinais de dificuldades iniciais no manejo da amamentação e correlacioná-los ao desmame precoce.

\section{MÉTODO}

Para cumprir os objetivos, foi conduzido um estudo prospectivo e observacional na Maternidade do Hospital Universitário da Fundação Universidade Federal de Mato Grosso do Sul (HU/FUFMS), cidade de Campo Grande, MS, durante o período de fevereiro de 2018 a junho de 2019. O hospital assiste em média 155 partos ao mês, exclusivamente a pacientes do Sistema Único de Saúde, e é referência estadual para Banco de Leite Humano em todo o Estado de Mato Grosso do Sul.

Todas as participantes concordaram em participar do estudo e assinaram o Termo de Consentimento Livre e Esclarecido. O respaldo ético foi obtido em comitê de ética institucional (Parecer 2.306.021).

A amostra foi por conveniência. O tamanho da amostra deste estudo foi determinado por uma calculadora on-line de tamanho de amostras. 0 cálculo amostral foi realizado considerando uma margem de erro de $5 \%$ e um nível de confiança de 95\%, com uma determinação de reposta de 50\%. Participaram do estudo 101 mães com idade acima de 18 anos, com parto único, em alojamento conjunto com seus recém-nascidos a termo (idade gestacional $\geq 37$ semanas) com peso ao nascer $\geq 10$ o percentil para gestação, de acordo com o gráfico de crescimento neonatal da Organização Mundial da Saúde (OMS) (VILLAR et al., 2014). A amamentação foi registrada na alta hospitalar que ocorreu dentro das primeiras $72 \mathrm{~h}$ após o parto. Os critérios de exclusão envolveram mães que não falam português ou pela perda de contato com a mãe, após a alta hospitalar.

\subsection{Instrumentos de avaliação}

As características sociodemográficas foram avaliadas por meio da ficha de avaliação elaborada pela pesquisadora responsável. A autoeficácia da amamentação foi avaliada por meio do questionário Breastfeeding 
Avaliação da mamada, autoeficácia do aleitamento materno e fatores influentes no desmame precoce em primíparas

Self-Efficacy Scale (BSES), versão completa, traduzido e validado na língua portuguesa como Escala de Autoeficácia da Amamentação, com o intuito de permitir a avaliação da confiança materna na amamentação, medindo a autoeficácia das mães na habilidade de amamentar (ORIÁ et al., 2009). São 33 questões divididas em dois domínios: Técnico e Pensamento Intrapessoal. Cada questão apresenta cinco possibilidades de resposta, com escores que variam de 1 a 5 , sendo: 1 - discordo totalmente; 2 - discordo; 3 - às vezes concordo; 4 - concordo; 5 - concordo totalmente. Assim, os escores totais da escala variam de 33 a 165 pontos. A autoeficácia na amamentação identificada pela escala é distribuída de acordo com as pontuações obtidas a partir da somatória de cada questão: autoeficácia baixa (33 a 118 pontos), autoeficácia média (119 a 137 pontos), autoeficácia alta (138 a 165 pontos).

A Escala Hospitalar de Ansiedade e Depressão (HAD) foi desenvolvida para identificar sintomas de ansiedade e de depressão em pacientes de hospitais clínicos não psiquiátricos, sendo posteriormente utilizada em outros tipos de pacientes, em pacientes não internados e em indivíduos sem doença. Um ponto importante que distingue a HAD das demais escalas é que, para prevenir a interferência dos distúrbios somáticos na pontuação da escala, foram excluídos todos os sintomas de ansiedade ou de depressão relacionados com doenças físicas (SILVA et al., 2016). A escala possui 14 itens, dos quais sete são voltados para a avaliação da ansiedade (HADS-A) e sete para a depressão (HADS-D). Cada um dos seus itens pode ser pontuado de zero a três, compondo uma pontuação máxima de 21 pontos para cada escala, adotados os pontos de cortes recomendados para ambas as subescalas.

Outro instrumento de avaliação utilizado foi o Formulário de Observação de Mamada preconizado pela OMS, cujo instrumento teve objetivo de avaliar a mamada e detectar possíveis problemas. Ele está dividido em seções da seguinte forma: Seção A - Observação geral (mãe/bebê); Seção B - Posição do bebê; Seção C - Pega; Seção D - Sucção.

Este instrumento possui opções que indicam sinais de uma amamentação bem-sucedida ou malsucedida. Logo, as marcações de sinais positivos indicam que o aleitamento materno está indo bem, caso contrário, se for 
observada a marcação de sinais negativos, a indicação é de que pode haver problemas na amamentação. A mamada foi classificada em boa, regular e ruim, seguindo o critério adotado, que se baseia no número de comportamentos sugestivos de dificuldades para cada aspecto avaliado (BRASIL, 2009).

Após o período de 6 meses da entrevista inicial, as mães foram contatadas por telefone a fim da obtenção das informações sobre a continuidade ou não da amamentação e a causa do desmame do bebê.

\subsection{Análise estatística}

As variáveis sociodemográficas, de autoeficácia da amamentação, ansiedade e depressão e a observação da mamada foram analisadas por ANOVA One-Way. Quando encontradas diferenças significativas $(p<0,05)$, foi realizado o teste post hoc de Student-Newman-Keuls de comparação múltipla para teste paramétrico ou o teste de Dunn's para distribuições não paramétricas. Em relação às variáveis qualitativas, quanto ao desfecho da amamentação e motivo do desmame precoce, elas foram analisadas usando o teste de Goodman para contrastes entre e dentro de populações multinomiais. Além disso, dados categóricos e contínuos foram analisados usando regressão logística binária em software Systat 13 (SYSTAT, Chicago, Illinois). Para todas as características internas, foram calculados odds ratio (OR) com intervalos de confiança (IC) de 95\% e o nível de significância considerado foi de 5\%.

\section{RESULTADOS}

A Tabela 1 apresenta algumas características sociodemográficas das 101 duplas mães/bebês. Observa-se que a amostra foi constituída, na sua maioria, por mulheres na faixa etária entre 18 e 25 anos, residentes na capital, em casa própria, que viviam em união estável, com renda familiar média de dois salários mínimos e amamentação exclusiva no pós-parto imediato. Quanto à religião, a maioria se declarou evangélica (38\%). A escolaridade materna foi de alta variância, apresentando maior proporção entre Ensino Médio completo (32\%) e incompleto em (29\%). Em relação ao trabalho, 55 (54\%) teriam direito ao salário-maternidade, apenas 36\% possuíam vínculo empregatício atual. Em relação aos dados pré-natais, houve uma variância 
Avaliação da mamada, autoeficácia do aleitamento materno e fatores influentes no desmame precoce em primíparas

de 1 a 20 no número de consultas realizadas, 48 (48\%) apresentaram intercorrências diversas e somente 46 (46\%) receberam orientações quanto à amamentação no período. Quanto aos dados perinatais, a média da idade gestacional ao nascimento foi de 39 semanas, sendo que a amostra se mostrou equilibrada quanto ao sexo do bebê e o tipo de parto, apresentando somente 1 (1\%) de parto gemelar.

Tabela 1- Características sociodemográficas e gestacionais das puérperas primíparas do Hospital-Escola da Universidade Federal de Mato Grosso do Sul, Campo Grande, MS, Brasil, 2017-2018

\begin{tabular}{lccc}
\hline Variáveis & $\mathbf{N}$ & $\%$ & Média (DP) \\
\hline Faixa etária & & & $23(5,3417)$ \\
$18-25$ & 74 & $73 \%$ & \\
$26-34$ & 21 & $21 \%$ & \\
$>34$ & 6 & $6 \%$ &
\end{tabular}

Origem de residência

Interior

$11 \quad 11 \%$

Capital

$9089 \%$

\section{Estado civil}

Solteira

$36 \quad 36 \%$

Casada

$26 \quad 26 \%$

União estável

$3939 \%$

\section{Religião}

Católica

$36 \quad 36 \%$

Evangélica

$38 \quad 38 \%$

Espírita

$33 \%$

Outros

$24 \quad 24 \%$

\section{Renda familiar (em salários mínimos)}

Até 1 salário mínimo

$24 \quad 24 \%$

Acima de 1 até 2 salários mínimos

$39 \quad 39 \%$

Acima de 2 até 3 salários mínimos

$23 \quad 23 \%$

Mais de 3 salários mínimos

$15 \quad 15 \%$

\section{Escolaridade materna}

Ensino Fundamental incompleto

$\begin{array}{cc}7 & 7 \% \\ 5 & 5 \% \\ 29 & 29 \%\end{array}$

Ensino Fundamental completo

Ensino Médio incompleto 
Ensino Médio completo

Ensino Superior incompleto

Ensino Superior completo

Pós-Graduação/Mestrado

Doutorado

Pós-Doutorado

Com vínculo empregatício

Direito ao salário-maternidade

\section{Condições de moradia}

Casa própria

Casa alugada

Casa cedida

Números de consultas de pré-natal

Variação

Orientações recebidas no pré-natal

Intercorrência na gestação

Idade gestacional ao nascer (em semanas)

Tipo de amamentação

Exclusiva

Mista

Tipo de parto

Vaginal

Cesáreo

Parto gemelar

Parto não gemelar

\section{Gênero do RN}

Masculino

Feminino

DP, desvio padrão.

Fonte: Autoria própria.

Na Tabela 2, pode-se observar que, em relação à Escala de Autoeficácia, houve prevalência de 78 (77\%) mães com alta confiança na amamentação. Quanto à Escala HADS-A, 23 (23\%) mães da amostra foram classificadas com ansiedade e 78 (77\%) sem ansiedade; na HADS-D, foram apontadas seis (6\%) com depressão e 95 (94\%) sem depressão. O desfecho apresentado quanto ao 
Avaliação da mamada, autoeficácia do aleitamento materno e fatores influentes no desmame precoce em primíparas

desmame precoce - antes dos 6 meses de idade do bebê - foi na proporção de $20(20 \%)$ do total da amostra. Entre os motivos do desmame, destaca-se o uso precoce do complemento (ao nascimento ou antes dos 6 meses), com 13 (13\%), e o fim da licença-maternidade, numa proporção de três (3\%).

Tabela 2- Índice da autoeficácia da amamentação, índice da escala hospitalar de ansiedade e depressão, índice e fatores que influenciaram no desmame precoce das puérperas primíparas do Hospital-Escola da Universidade Federal de Mato Grosso do Sul, Campo Grande, MS, Brasil, 2017-2018

\begin{tabular}{lccc}
\hline Variáveis & N & \% & Média (DP) \\
\hline Escala de Autoeficácia & 78 & $77 \%$ & \\
Alta & 20 & $20 \%$ & \\
Média & 3 & $3 \%$ & $\mathbf{5 , 7 8 ( 3 , 5 5 )}$ \\
Baixa & & & \\
Pontuação de ansiedade & 23 & $23 \%$ & \\
Com ansiedade & 78 & $77 \%$ & $\mathbf{4}, \mathbf{9 3} \mathbf{( 2 , 0 0 )}$ \\
Sem ansiedade & & & \\
Pontuação de depressão & 6 & $6 \%$ & \\
Com depressão & 95 & $94 \%$ & \\
Sem depressão & $\mathbf{2 0}$ & $\mathbf{2 0} \%$ & \\
Desmame antes de 6 meses & & & \\
Motivo de desmame & 13 & $13 \%$ & \\
Utilização precoce do complemento & 3 & $3 \%$ & \\
Fim da licença-maternidade & 2 & $2 \%$ & \\
Internação da mãe ou do bebê & 1 & $1 \%$ & \\
Alergia alimentar & 1 & $1 \%$ & \\
Introdução alimentar precoce &
\end{tabular}

DP, desvio padrão.

Fonte: Autoria própria.

Na Tabela 3, a observação da mãe na Escala de Observação da Mamada constituiu a variável mais preditiva para ocorrência do desfecho com desmame precoce entre as puérperas primíparas $(p<0,05)$; o modelo de registros logísticos binários destinado a apresentar associação entre fatores de risco revelou significância estatística em prever o desmame precoce em primíparas. 
Tabela 3 - Coeficientes de regressão logística binária para dados sociodemográficos, classificação das escalas de autoeficácia na amamentação, ansiedade e depressão e observação da mamada

\begin{tabular}{|c|c|c|c|c|c|}
\hline \multirow{3}{*}{ Fator de risco } & \multicolumn{5}{|c|}{ Regressão logística } \\
\hline & \multirow{2}{*}{ Coeficiente } & \multirow{2}{*}{ OR } & \multicolumn{2}{|c|}{ IC (95\%) } & \multirow{2}{*}{ p-valor } \\
\hline & & & L. Inf. & L. Sup. & \\
\hline Constante & 59,100 & - & - & - & 0,000 \\
\hline Idade & 0,063 & 1,065 & 0,922 & 1,230 & 0,392 \\
\hline Estado civil & 0,056 & 1,058 & 0,469 & 2,387 & 0,892 \\
\hline Renda & 0,029 & 1,029 & 0,469 & 2,257 & 0,943 \\
\hline Escolaridade & $-0,248$ & 0,780 & 0,430 & 1,415 & 0,414 \\
\hline V. E. & $-0,354$ & 0,702 & 0,150 & 3,279 & 0,653 \\
\hline N. C. P. & $-0,066$ & 0,936 & 0,748 & 1,170 & 0,560 \\
\hline R. O. S. A. P. & $-0,924$ & 0,397 & 0,092 & 1,709 & 0,215 \\
\hline Tipo de parto & 0,648 & 1,911 & 0,416 & 8,775 & 0,405 \\
\hline P. E. A. E. A. & $-0,055$ & 0,946 & 0,847 & 1,058 & 0,331 \\
\hline C. E. A. E. A. & 1,042 & 2,835 & 0,222 & 36,290 & 0,423 \\
\hline P. Ansiedade & $-0,166$ & 0,847 & 0,668 & 1,074 & 0,171 \\
\hline P. Depressão & 0,316 & 1,372 & 0,896 & 2,100 & 0,146 \\
\hline E. O. M. - Mãe & 2,418 & 11,221 & 2,773 & 45,406 & $0,001 *$ \\
\hline E. O. M. - Bebê & $-26,364$ & 0,000 & 0,000 & 0,000 & - \\
\hline E. O. M. - Pos. & 0,022 & 1,022 & 0,150 & 6,982 & 0,982 \\
\hline E. O. M. - Pega & $-0,135$ & 0,874 & 0,053 & 14,305 & 0,925 \\
\hline E. O. M. - Suc. & $-0,984$ & 0,374 & 0,043 & 3,227 & 0,371 \\
\hline \multicolumn{6}{|c|}{$\begin{array}{l}\text { OR, odds ratio; IC, intervalo de confiança; L. Inf., limite inferior; L. Sup., limite superior; V. E., } \\
\text { vínculo empregatício; N. C. P., número de consultas pré-natal; R. O. S. A. P., recebeu orien- } \\
\text { tações sobre aleitamento no pré-natal; P. E. A. E. A., pontuação em Escala de Autoeficácia } \\
\text { na Amamentação; C. E. A. E. A., classificação de Escala de Autoeficácia na Amamentação; } \\
\text { P. Ansiedade, pontuação de ansiedade; P. Depressão, pontuação de depressão; E. O. M. } \\
\text { - Mãe, Escala de Observação da Mamada - observação da mãe; E. O. M. - Bebê, Escala } \\
\text { de Observação da Mamada - observação do bebê; E. O. M. - Pos., Escala de Observação } \\
\text { da Mamada - observação da posição do bebê; E. O. M. - Pega, Escala de Observação da } \\
\text { Mamada - observação da pega; E. O. M. - Suc., Escala de Observação da Mamada - ob- } \\
\text { servação da sucção; * p< } 0,05 \text {. }\end{array}$} \\
\hline
\end{tabular}

Fonte: Autoria própria.

Na Tabela 4, pode-se observar a classificação da mamada em Boa, Regular ou Ruim, levando em consideração a quantidade de comportamentos 
Avaliação da mamada, autoeficácia do aleitamento materno e fatores influentes no desmame

indicativos de dificuldade. Logo, a maioria das puérperas (90\%) não apresentou dificuldades na amamentação e obteve a classificação da mamada como Boa, com destaque aos comportamentos referentes à observação geral do bebê, posição do bebê e pega. Entre os comportamentos, a observação geral da mãe apresentou dificuldades, com maior quantificação na classificação Ruim (6\%).

Tabela 4 - Classificação das mamadas segundo comportamentos observados, Maternidade/HUMAP, Campo Grande, MS, Brasil, 2017-2018

\begin{tabular}{lcccccc}
\hline \multirow{2}{*}{\multicolumn{1}{c}{ Comportamento }} & \multicolumn{2}{c}{ Boa } & \multicolumn{2}{c}{ Regular } & \multicolumn{2}{c}{ Ruim } \\
\cline { 2 - 7 } & $\mathbf{N}$ & $\mathbf{\%}$ & $\mathbf{N}$ & $\mathbf{\%}$ & $\mathbf{N}$ & $\%$ \\
\hline Observação geral - mãe & 80 & 79 & 15 & 15 & 6 & 6 \\
Observação geral - bebê & 100 & 99 & 1 & 1 & 0 & 0 \\
Posição do bebê & 94 & 93 & 6 & 6 & 1 & 1 \\
Pega & 95 & 94 & 5 & 5 & 1 & 1 \\
Sução & 88 & 87 & 11 & 11 & 2 & 2 \\
Total & 457 & 90 & 38 & 8 & 10 & 2 \\
\hline
\end{tabular}

Fonte: Autoria própria.

A Tabela 5 apresenta a quantidade de comportamentos indicativos de dificuldade para cada aspecto avaliado na observação da mamada, os quais totalizaram 211 comportamentos. Destes, 75 (35,5\%) referem-se à observação geral da mãe como maior aspecto de dificuldade avaliado, com destaque à presença de mamas avermelhadas, inchadas e/ou doloridas e mamas seguradas com dedos na aréola. Outro aspecto apresentado como desfavorável é a sucção, com 70 (33,2\%) comportamentos indicando dificuldade na mamada, com prevalência dos sinais do reflexo da oxitocina não percebidos e do momento quando a mãe tira o bebê da mama. Quanto à posição do bebê, embora apresentada como baixa dificuldade, com 28 $(13,3 \%)$ dos comportamentos desfavoráveis, destaca-se o posicionamento do pescoço/cabeça do bebê girados ao mamar. Da mesma forma, na observação geral do bebê, com apenas 11 (5,2\%) comportamentos que indicam dificuldade, prevaleceu o bebê inquieto ou chorando e o apoio frágil com falta de contato visual entre mãe e bebê. 
Tabela 5 - Distribuição dos comportamentos indicativos de dificuldade na observação da mamada, segundo aspectos avaliados, Maternidade/HUMAP, Campo Grande, MS, Brasil, 2017-2018

\begin{tabular}{|c|c|c|}
\hline \multirow{2}{*}{ Aspectos avaliados } & \multicolumn{2}{|c|}{ Comportamentos } \\
\hline & $\mathbf{N}$ & $\%$ \\
\hline \multicolumn{3}{|l|}{ Observação geral - mãe } \\
\hline Mãe parece doente ou deprimida & 3 & 1,4 \\
\hline Mãe parece tensa e desconfortável & 12 & 5,7 \\
\hline Mamas avermelhadas, inchadas e/ou doloridas & 41 & 19,4 \\
\hline Mama segurada com dedos na aréola & 19 & 9,0 \\
\hline Subtotal & 75 & 35,5 \\
\hline \multicolumn{3}{|l|}{ Observação geral - bebê } \\
\hline Bebê parece sonolento ou doente & 1 & 0,5 \\
\hline Bebê inquieto ou chorando & 6 & 2,8 \\
\hline Sem contato visual entre mãe e bebê, apoio frágil & 4 & 1,9 \\
\hline O bebê não busca nem alcança & 0 & 0 \\
\hline Subtotal & 11 & 5,2 \\
\hline \multicolumn{3}{|l|}{ Posição do bebê } \\
\hline Pescoço/cabeça do bebê girados ao mamar & 15 & 7,1 \\
\hline Bebê não é seguro próximo ao corpo da mãe & 3 & 1,4 \\
\hline Queixo e lábio inferior opostos ao mamilo & 8 & 3,8 \\
\hline Bebê não apoiado & 2 & 0,9 \\
\hline Subtotal & 28 & 13,3 \\
\hline \multicolumn{3}{|l|}{ Pega } \\
\hline Mais aréola é vista abaixo do lábio inferior & 3 & 1,4 \\
\hline A boca do bebê não está bem aberta & 12 & 5,7 \\
\hline Lábios voltados para frente/virados para dentro & 10 & 4,7 \\
\hline O queixo do bebê não toca a mama & 2 & 0,9 \\
\hline Subtotal & 27 & 12,8 \\
\hline \multicolumn{3}{|l|}{ Sucção } \\
\hline Sucções rápidas e superficiais & 11 & 5,2 \\
\hline Mãe tira o bebê da mama & 21 & 10,0 \\
\hline Sinais do reflexo da oxitocina não percebidos & 28 & 13,3 \\
\hline Mamas parecem duras e brilhantes & 10 & 4,7 \\
\hline Subtotal & 70 & 33,2 \\
\hline Total & 211 & 100,0 \\
\hline
\end{tabular}

Fonte: Autoria própria. 
Avaliação da mamada, autoeficácia do aleitamento materno e fatores influentes no desmame precoce em primíparas

\section{DISCUSSÃO}

A OMS e o UNICEF empreendem esforços para proteger, promover e apoiar o aleitamento materno exclusivo (AME), para que as mães possam estabelecer e mantê-lo pela primeira vez até os seis meses de vida do bebê (BRASIL, 2009). Mas essa realidade no Brasil ainda está longe de ser alcançada, uma vez que a prevalência de aleitamento materno exclusivo nos primeiros 180 dias, nas regiões brasileiras, é de apenas 63\%, 59\%, 51\%, 61\% e $56 \%$, respectivamente, para Norte, Nordeste, Sudeste, Sul e Centro-Oeste. Na área rural, foi de 60\% e, na urbana, 58\% (WENZEL; SOUZA, 2011). Tal fato nos guiou a estudar os motivos do desmame precoce em primíparas. Mais especificamente, as dificuldades iniciais durante a amamentação, observadas pelo profissional de saúde, e sua associação ao desmame precoce, além da correlação da percepção da mãe, por meio do escore da Escala de Autoeficácia do Aleitamento Materno, com o aleitamento materno exclusivo em primíparas.

Em relação ao perfil das primíparas assistidas pela Maternidade do Hospital-Escola, verificou-se que o perfil das participantes do estudo é de mulheres com idade entre 18 e 25 anos, residentes na capital, em casa própria, com união estável, renda familiar com média de até dois salários mínimos, em amamentação exclusiva no pós-parto imediato, religiosas cristãs evangélicas, com escolaridade de Ensino Médio incompleto prevalecente. Um terço delas possui vínculo empregatício e pouco mais da metade tem direito à licença-maternidade. Houve uma média de 18 consultas pré-natais, embora tenha havido alta variância. Aproximadamente metade da amostra recebeu orientações sobre a amamentação no pré-natal e apresentou diversas intercorrências sem gravidade. A média da idade gestacional ao nascimento foi de 39 semanas, com sexo do recém-nascido e tipo de parto equiparados. A amostra apresentou elevado escore de autoeficácia na amamentação. E, em relação à saúde mental, verificou-se que $23 \%$ apresentaram escore compatível com sintomas de ansiedade e $6 \%$ com os de depressão. Um quinto desta população obteve o desfecho de desmame precoce devido, principalmente, segundo relatado pelas mães, ao uso precoce do complemento e o fim da licença-maternidade. 
A disparidade entre as mulheres que possuíam vínculo empregatício ativo e as que receberiam o salário-maternidade se dá pelo benefício previsto no art. 201, II da Constituição Federal, nos artigos 71 e seguintes da Lei 8.213/91 e nos artigos 93 e seguintes do Decreto 3.048/99, os quais afirmam que o salário-maternidade é devido a todas as seguradas da Previdência Social, gestantes ou adotantes, sejam elas empregadas, avulsas, domésticas, contribuintes especiais, facultativas ou individuais, sejam elas desempregadas; desde que a segurada esteja dentro do período de graça (12 meses a contar da última contribuição) e tiver cumprido a carência (10 meses de contribuição), ela tem direito ao salário-maternidade. A concessão do salário maternidade agiu positivamente nessa amostra, ampliando a condição financeira das mulheres, considerando um estudo que indica que a adesão à amamentação responde positivamente a um melhor nível de renda familiar (GIGANTE; VICTORA; BARROS, 2000).

Na análise do escore de autoeficácia, não houve correlação significativa entre a percepção da mãe e o desmame precoce. Na verdade, o grau de autoeficácia verificado neste estudo foi elevado, com poucas respostas desfavoráveis. Isso pode indicar que grande parte das mães tem alta confiança e boa percepção sobre a amamentação. É muito provável que esse dado se refira à boa qualidade do suporte ao aleitamento, oferecido pelos profissionais do serviço às mulheres, durante a permanência na maternidade. Por se tratar de um hospital de referência regional para Banco de Leite Humano, dispõe de um atendimento especializado no manejo da lactação, realizado por profissionais treinados.

Há de se ressaltar, também, o fato de que seja socialmente aceito dar respostas favoráveis, sendo muito ameaçador para a mãe iniciar o seu discurso enfatizando os aspectos ruins sentidos por ela, que são tão negados pela sociedade. Logo, esse fator deve ser de relevância na atenção dos profissionais de saúde que incentivam o aleitamento materno, para estarem atentos ao sofrimento, ao cansaço e, enfim, serem receptivos às dificuldades relatadas pelas mulheres ao amamentar (WILLIAMSON et al., 2012).

Um estudo mostra uma análise que esclarece quão problemático pode ser para as mulheres terem dificuldades em amamentar em uma cultura que, pelo menos em parte, sustenta uma abordagem romantizada da prática, 
Avaliação da mamada, autoeficácia do aleitamento materno e fatores influentes no desmame precoce em primíparas

especialmente se elas percebem suas dificuldades como sinal de desvio ou inadequação pessoal. Logo, esse sentimento de inadequação ou vergonha do "fracasso" de amamentar pode tornar menos provável que algumas mulheres procurem ajuda para superar as dificuldades na amamentação e menos propensas a falar abertamente sobre elas ou a explorar soluções para os desafios que enfrentam (ALKOZEI; MCMAHON; LAHAV, 2014).

Um estudo recente avaliou a relação entre o estresse da mãe e a amamentação e demonstrou que o ato ou a atitude de amamentar diminui à medida que o estresse percebido pela mãe aumenta. Sendo assim, as mães devem aprender estratégias sobre como lidar com o estresse, a fim de melhorar seu engajamento em relação à amamentação, e que serviços de aconselhamento devem ser iniciados no pré-natal, a fim de incentivá-las a amamentar (DURAN; KAYNAK; KARADAS, 2019).

No presente estudo, foi observado que $23 \%$ das mães apresentaram escores compatíveis com ansiedade e $6 \%$ com depressão. Estes índices foram consideravelmente baixos se comparados a outros na literatura, em que 52\% das mães experimentaram aumento do estresse após o nascimento. Uma revisão sistemática sobre o assunto concluiu que o efeito da ansiedade no período pós-parto diminui a autoeficácia, aumenta as dificuldades e pode afetar negativamente os comportamentos da amamentação e a composição do leite materno (FALLON et al., 2016); e sugere, ainda, que mulheres com ansiedade pós-parto têm menor probabilidade de amamentação exclusiva e maior probabilidade de suplementar com fórmula no hospital (MATTHEY; FISHER; ROWE, 2013).

Os fatores que influenciam o desmame precoce, segundo o relato das mães deste estudo, foram o uso do complemento precocemente (ao nascimento ou antes dos 6 meses) e o fim da licença-maternidade. A introdução precoce de outros alimentos pela mãe contribui para o desmame precoce, o que, por sua vez, interfere na dinâmica de sucção e extração do leite materno, uma vez que, saciado, o bebê suga menos, o que leva ao deficit do esvaziamento da mama e consequentemente diminui a produção do leite da mãe (FLAHERMAN et al., 2019).

Outros trabalhos apontam que a presença de sintomas psicológicos no pós-parto aumenta as chances de dificuldades da díade mãe/bebê na 
amamentação e diminui a confiança materna para amamentar, além de elevar a probabilidade de interrupção do AME (ISLAM et al., 2017).

Em um estudo recente, foram investigadas, nos primeiros meses após o parto, as dificuldades de amamentação vivenciadas por mães de bebês saudáveis nascidos a termo e sua associação com a descontinuação precoce da amamentação; conclui-se que o retorno ao trabalho esteve associado à falha precoce do aleitamento materno exclusivo, pois equilibrar o trabalho e a amamentação exclusiva é desafiador e requer um forte apoio em curto e longo prazo (GIANNI et al., 2019).

Neste estudo, foram detectadas altas frequências de parâmetros de três ou quatro comportamentos desfavoráveis à amamentação, relacionados aos aspectos gerais observados quanto ao posicionamento mãe/bebê, de acordo com o protocolo de observação da mamada.

É recente o conhecimento de que o posicionamento adequado do binômio mãe/bebê e a pega efetiva favorecem a prática do aleitamento exclusivo; portanto uma posição inadequada, como segurar as mamas com os dedos em tesoura sobre a aréola, dificulta o posicionamento da boca do bebê em relação ao mamilo e pode resultar em má pega (INGRAN; JOHNSON; GREENWOOD, 2002). Estudos ultrassonográficos mostram que, quando o bebê tem pega adequada, o mamilo fica posicionado na parte posterior do palato, protegido da fricção e compressão, o que previne traumas mamilares (GEDDES et al., 2017).

Em um estudo, foi apresentada a eficácia de uma orientação sobre a técnica adequada de amamentação na maternidade como estratégia para diminuir a frequência de mulheres que referem baixa produção de leite (ALATALO et al., 2019). Contudo a pega inadequada causa lesões mamilares, o que pode resultar em dor e desconforto à mãe, comprometendo a prática do aleitamento materno de forma contínua, caso esse problema não seja corrigido (DEGEFA et al., 2019).

Este estudo não fez distinção se o aleitamento aos seis meses era complementar ou exclusivo, entretanto um estudo sobre prevalência do aleitamento em nossa região demonstrou que apenas 14,4\% das mães realizavam aleitamento exclusivo até os seis meses, e a introdução alimentar 
Avaliação da mamada, autoeficácia do aleitamento materno e fatores influentes no desmame precoce em primíparas

ocorreu antes do sexto mês na maioria dos casos (KAMIYA et al., 2019). Considerando que as primíparas mantiveram o bebê ao seio materno até os 6 meses, exclusivamente ou de forma complementar, o percentual de desmame precoce encontrado foi moderadamente baixo.

Este trabalho também mostrou que se faz necessário o olhar do profissional da saúde em identificar as dificuldades na prática da amamentação, pois, mesmo com altos níveis de autoeficácia pela perspectiva materna, foi apontado que o fator de maior relevância para o desmame precoce está relacionado à observação da condição física da mãe pelo profissional treinado. Portanto é cabível que uma maior disponibilidade de tempo da equipe em apoiar as mães nas primeiras mamadas poderá proporcionar uma correção precoce das principais dificuldades inerentes à lactação.

\subsection{Limitações}

Este estudo teve alguns cuidados metodológicos, tais como o treinamento das pesquisadoras responsáveis pelas avaliações de mamadas, até que atingissem alta concordância em todos os parâmetros avaliados. O fato de as pesquisadoras que realizaram as avaliações das mamadas não terem vínculo com o hospital em que se realizou a pesquisa e de as avaliações na maternidade e por contato telefônico para cada dupla mãe/bebê serem realizadas pela mesma pesquisadora certamente contribuiu para a minimização de um possível viés de aferição. O ideal seria se as avaliações de mamadas fossem feitas por duas observadoras independentes, mas não dispúnhamos de recursos humanos suficientes para tal.

Conquanto vislumbremos qualidades do estudo, as suas limitações não devem ser negligenciadas. Em primeiro lugar, é importante destacar que foram incluídas no estudo apenas participantes que estavam vivenciando a amamentação pela primeira vez, o que restringiu a amostra. A exclusão de participantes que amamentaram outros filhos se deu pelo fato de que a amamentação possui menos eventos desfavoráveis quando a mãe já teve uma experiência prévia, o que deixaria a amostra heterogênea. Além disso, as pacientes com bebês prematuros e outras comorbidades associadas ao binômio materno fetal poderiam causar viés sobre os resultados. 
Em segundo lugar, devemos enfatizar que, na prática clínica, o engajamento das mães na amamentação nem sempre é similar entre todas elas, devido a diferentes condições psicossociais e redes de apoio que a puérpera encontra em seu ambiente social e familiar. Neste caso, torna-se fundamental o monitoramento das primíparas pelo profissional de saúde, a fim de realizar uma avaliação ampla, enfatizando os parâmetros desfavoráveis à amamentação presentes em cada caso.

\section{CONCLUSÃO}

O presente estudo demonstrou que houve correlação entre o desmame precoce e a condição física da mãe no momento da observação da mamada. Essas dificuldades iniciais encontradas são comportamentos preditivos para o desmame precoce: mamas avermelhadas, inchadas e/ou doloridas, apoio da mama com dedos em tesoura sobre a aréola e o nível de tensão ou desconforto da mãe no momento da amamentação. Tal fato reforça a importância da avaliação e intervenção precoce do profissional de saúde, a fim de oferecer suporte à nutriz, no que diz respeito às possíveis dificuldades encontradas no primeiro aleitamento, e evitar o desmame precoce.

\section{REFERÊNCIAS}

ALKOZEI, A.; MCMAHON, E.; LAHAV, A. Stress levels and depressive symptoms in NICU mothers in the early postpartum period. The Journal of Maternal-Fetal \& Neonatal Medicine, v. 27, n 17, p. 1738-43, nov. 2014.

ALATALO, D.; JIANG, L.; GEDDES, D.; HASSANIPOUR, F. Capturing nipple deformation and peripheral pressure on the areola during breastfeeding. Journal Biomechanical Engineering, v. 142, n. 1, maio 2019. DOI: https://doi.org/10.1115/1.4043665

BRASIL. Ministério da Saúde (BR), Secretaria de Atenção à Saúde. Departamento de Atenção Básica. Saúde da criança: nutrição infantil: aleitamento materno e alimentação complementar. Brasília, DF, 2009.

DEGEFA, N.; TARIKU, B.; BANCHA, T.; AMANA, G.; HAJO, A.; KUSSE, Y.; ZERIHUN, E.; ASCHALEW, Z. Breast feeding practice: positioning and attachment during breast feeding among lactating mothers visiting health facility in Areka Town, Southern 
Avaliação da mamada, autoeficácia do aleitamento materno e fatores influentes no desmame precoce em primíparas

Ethiopia. International Journal of Pediatrics, v. 2019, ID 8969432, abr. 2019. DOI: https://doi.org/10.1155/2019/8969432

DURAN S.; KAYNAK, S.; KARADAS, A. The relationship between breastfeeding attitudes and perceived stress levels of Turkish mothers. Scandinavian Journal of Caring Sciences, 2019.

FALLON, V.; GROVES, R.; HALFORD, J. C.; BENNETT, K. M.; HARROLD, J. A. Postpartum anxiety and infant-feeding outcomes. Journal of Human Lactation, v. 32, n. 4, p. 740-58, nov. 2016. DOI: https://doi.org/10.1177/0890334416662241

FLAHERMAN, J. V.; MCKEAN, M.; BRAUNREUTHER, E.; KAIR, L. R.; CABANA, M. D. Minimizing the relationship between early formula use and breastfeeding cessation by limiting formula volume. Breastfeeding Medicine, v. 14, n. 8, p. 533-37, out. 2019. DOI: https://doi.org/10.1089/bfm.2019.0055

GEDDES, D. T.; CHOOI, K.; NANCARROW, K.; HEPWORTH, A. R.; GARDNER, H.; SIMMER, K. Characterisation of sucking dynamics of breastfeeding preterm infants: a cross sectional study. BMC Pregnancy and Childbirth, v. 17, n. 1, p. 386, nov. 2017. DOI: https://doi.org/10.1186/s12884-017-1574-3

GIANNI, M. L. et al. Breastfeeding difficulties and risk for early breastfeeding cessation. Nutrients, v. 11, n. 10, PII: E2266, set. 2019. DOI: https://doi.org/10.3390/ nu11102266

GIGANTE, D. P.; VICTORA, C. G.; BARROS, F. C. Nutrição materna e duração da amamentação em uma coorte de nascimento de Pelotas, RS. Revista de Saúde Pública, São Paulo, v. 34, n. 3, p. 259-65, 2000.

INGRAM, J.; JOHNSON, D.; GREENWOOD, R. Breastfeeding in Bristol: teaching good positioning, and support from fathers and families. Midwifery, v. 18, n. 2, p. 87-10, jun.2002.

ISLAM, M. J.; BAIRD, K.; MAZEROLLE, P.; BROIDY, L. Exploring the influence of psychological factors on exclusive breastfeeding in Bangladesh. Archives of Women's Mental Health, v. 20, n. 1, p. 173-88, fev. 2017. DOI: https://doi.org/10.1007/ s00737-016-0692-7

KAMIYA, E.; MENDONÇA, L. A. B. M.; FERREIRA, R. S., PALHARES, D. B. Prevalência de aleitamento materno em Campo Grande, Mato Grosso do Sul, Brasil. Multitemas, Campo Grande, MS, v.24, 57, p. 257-72, maio/ago. 2019. 
MATTHEY, S.; FISHER, J.; ROWE, H. Using the Edinburgh postnatal depression scale to screen for anxiety disorders: conceptual and methodological considerations. Journal of Affective Disorders, v. 146, n. 2, p. 224-30, abr. 2013. DOI: https://doi. org/10.1016/j.jad.2012.09.009

ORIA, M. O.; XIMENES, L. B.; DE ALMEIDA, P. C.; GLICK, D. F.; DENNIS, C.-L. Psychometric assessment of the Brazilian version of the Breastfeeding Self-Efficacy Scale. Public Health Nursing, v. 26, n. 6, p. 574-83, nov./dez. 2009. DOI: https:// doi.org/10.1111/j.1525-1446.2009.00817.x

SILVA, H. A.; PASSOS, M. H. P.; OLIVEIRA, V. M. A.; PALMEIRA, A. C.; PITANGUI, A. C. R.; ARAÚJO, R. C. Short version of the Depression Anxiety Stress Scale-21: is it valid for Brazilian adolescents? Einstein (São Paulo), v. 14, n. 4, p. 486-93, out./dez. 2016. DOI: https://doi.org/10.1590/s1679-45082016ao3732

VILLAR, J. et al. International standards for newborn weight, length, and head circumference by gestational age and sex: the Newborn Cross-Sectional Study of the INTERGROWTH-21st Project. The Lancet, v. 384, n. 9946, p. 857-68, set. 2014. DOI: https://doi.org/10.1016/S0140-6736(14)60932-6

WENZEL, D.; SOUZA, S. B. Prevalência do aleitamento materno no Brasil segundo condições socioeconômicas e demográficas. Journal of Human Growth and Development, v. 21, n. 2, p. 251-8, 2011. DOI: https://doi.org/10.7322/jhgd.20013.

WILLIAMSON, I.; LEEMING, D.; LYTTLE, S.; JOHNSON, S. 'It should be the most natural thing in the world': exploring first-time mothers' breastfeeding difficulties in the UK using audio-diaries and interviews. Maternal \& Child Nutrition, v. 8, n. 4, p. 434-47, out. 2012. 